Chadwick, P. (1959). J. gen. Microbiol. 21, 631-634

\title{
Rapid identification of Bacillus anthracis by Microscopical Observation of Bacteriophage Lysis
}

\author{
By P. CHADWICK* \\ Microbiological Research Establishment, Porton, Wiltshire
}

\begin{abstract}
SUMMARY: When young growing filaments or microcolonies of Bacillus anthracis were treated with specific bacteriophage, fragmentation of the filaments followed by complete disintegration of the microcolony were visible microscopically within 2-3 hr. Other Bacillus species were unaffected by the phage. This technique is a simple one for accelerating the identification of Bacillus anthracis.
\end{abstract}

Bacillus anthracis may be distinguished from the closely related $B$. cereus by its susceptibility to a specific bacteriophage (McCloy, 1951). The usual technique for testing a suspected strain with phage consists in spotting the undiluted phage on a bacterial lawn on agar, and observing for lysis after incubation overnight (McCloy, 1951; Brown \& Cherry, 1955). It seemed that identification could be accelerated by applying the phage to the bacteria at an earlier stage of their growth, and observing lysis microscopically. The experiments reported in this paper confirmed this prediction.

\section{METHODS AND RESULTS}

Bacteriophage. The bacteriophage used was a virulent mutant $\alpha$ of a temperate phage $\beta$, derived from a Bacillus cereus strain W (McCloy, 1958). The phage was used undiluted at a titre of $1.5 \times 10^{10}$ particles $/ \mathrm{ml}$. The phage may be propagated on any strain of $B$. anthracis.

Propagation technique. A $\mathbf{1 0}^{-2}$ dilution in broth of an overnight culture of Bacillus anthracis was incubated for $3 \mathrm{hr}$. at $37^{\circ}$. The culture tube was held in a Perspex drum rotating at about 2 rev./sec., inclined at $45^{\circ}$ and fixed in a wooden box, the temperature inside which was controlled thermostatically. The drum was rotated by a low-power motor. The source of heat was a $100 \mathrm{~W}$. electric light bulb placed beneath a metal reflector. To the resulting logarithmic phase culture was added $\alpha$ phage in the proportion of $10^{9}$ particles $/ 10 \mathrm{ml}$. culture, and incubation continued until clearing was complete, usually about $2 \frac{1}{2} \mathrm{hr}$. after addition of the phage. The bacterial debris was centrifuged, and the supernatant liquid treated with $1 \mathrm{ml}$. chloroform. The lysate was left in contact with chloroform for $30 \mathrm{~min}$. on the bench, and the chloroform then removed by suction. A sterility test was performed on the lysate, which was afterwards ready for use.

Technique of the phage-lysis microtest. Light inocula of Bacillus anthracis

* Present address : Department of Bacteriology, London School of Hygiene and Tropical Medicine, Keppel Street (Gower Street), London, W.C.1. 
spores were allowed to germinate on plates of nutrient agar, with or without $5 \%(\mathrm{v} / \mathrm{v})$ peptic extract of sheep blood (PSB) added. After 4-5 hr. incubation at $37^{\circ}$, single strand filaments of $B$. anthracis were visible under a binocular dissecting microscope, or a monocular microscope with transmitted light and $25 \mathrm{~mm}$. or $16 \mathrm{~mm}$ : objectives. The filaments elongated rapidly in the early phase of growth, frequently reaching a length of 250-500 $\mu$ in one dimension before showing any tendency to form loops or folds. A single loop near the mid-point was, however, often characteristic. Plate 1, fig. 1, shows typical filaments of $B$. anthracis growing on nutrient agar inoculated $4 \mathrm{hr}$. previously with a light spore suspension. Plate 1, fig. 2, shows a microcolony of the same strain after growth for $6 \mathrm{hr}$. on nutrient agar.

The position of the filament on the agar surface was marked with a straight wire: A small loopful of undiluted $\alpha$ phage was applied to the area of agar carrying the filament, and allowed to soak into it. There was some advantage in watching the application of the phage through the microscope to note any mechanical disturbance of the filament. It was also desirable to examine the treated area microscopically after the liquid had dried in, to ensure that the filament had not been removed accidentally on the loop. The risk of this happening was small.

The plate was returned to the $37^{\circ}$ incubator for about $1 \mathrm{hr}$. At this time, and on several occasions during the subsequent hour, the treated area was examined microscopically for evidence of lysis in the filament. Between examinations the plate was returned to the incubator or kept in a hot-box $\left(37^{\circ}\right)$ equipped with a microscope. During the second hour after application of $\alpha$ phage, breaks appeared in the filament, due to the action of phage, and at about the end of this second hour, the filament had been completely lysed, and was represented by a thin groove in the agar corresponding to the position of the original filament (Pl. 1, figs. 3, 4). If the phage was applied to a filament at a slightly later stage of development than that shown in fig. 1 , or to. a microcolony such as that shown in fig. 2, then growth continued for another 1-1. $\mathrm{t}$ hr. before fragmentation began. Disintegration of the microcolony was, however, complete within $3 \mathrm{hr}$. of application of the phage.

optical apparatus. The lytic changes were observed by using a monocular microscope with 16 and $4 \mathrm{~mm}$. objectives and $\times 10$ ocular, the source of illumination being transmitted light from a high-power filament lamp. The vertical illumination method described by Pearce \& Powell (1951) was also tried. Observations were made with (i) a $16 \mathrm{~mm}$. objective, using a light background; (ii) a $4 \mathrm{~mm}$. objective, using a dark background.

Under the $16 \mathrm{~mm}$. objective, filaments of Bacillus anthracis showed alternating light and dark areas due to parts of the filament being embedded in the agar while other segments lay flat on the surface. During the action of the phage, the grooves left in the agar by the lysed portions of the filaments contrasted strongly with the segments not yet lysed. But vertical illumination with this objective was not suitable for observation of fine detail, because of the 'frosted' appearance of the agar after application of the phage. By transmitted light the background was relatively clear. 
The dark background for use with the $4 \mathrm{~mm}$. objective was provided by incorporating nigrosin $0.5 \%(\mathrm{w} / \mathrm{v})$ in the medium. When bacterial growth and phage lysis were allowed to take place on this medium, examination with the $4 \mathrm{~mm}$. objective revealed very striking patterns of lysis. The irregular distribution of damage by the phage was very obvious, and when observation was continuous, individual cells could be seen to 'explode' as lysis took place.

Reproducibility of phage-lysis with different strains of Bacillus anthracis. Microcolonies of 13 virulent $B$. anthracis strains were allowed to develop from light spore inocula on nutrient agar, and one colony of each strain tested with $\alpha$ phage. Signs of disintegration of the colony were evident with each strain within $3 \mathrm{hr}$. of application of the phage.

Tests with other species of the genus Bacillus. The $\alpha$-phage caused no damage to filaments or microcolonies of one strain each of Bacillus cereus, B. mycoides, $B$. licheniformis or $B$. subtilis, when these were tested in parallel with strains of $B$. anthracis. Plate 1 , fig. 5, shows a microcolony of $B$. cereus after $4 \mathrm{hr}$. incubation on nutrient agar. Plate 1 , fig. 6, shows a larger microcolony of $B$. cereus which had been treated with $\alpha$-phage after $4 \mathrm{hr}$. incubation and then incubated for a further $1 \frac{1}{2} \mathrm{hr}$.

Other control measures. When $\alpha$-phage was applied to a filament or microcolony, a similar filament or microcolony on the same plate was treated with a loop of sterile broth in the same way. Such control filaments always grew normally into microcolonies which never showed any fragmentation of their constituent filaments such as might have been produced by mechanical means.

Total time for identification of Bacillus anthracis by the phage-lysis microtest. The time elapsing between inoculation of agar with a light spore suspension and the observation of convincing lysis of the resulting microcolonies was 5-7 hr. The shorter times were observed on medium enriched with PSB, where growth and lysis were slightly faster than on plain nutrient agar.

\section{DISCUSSION}

The specificity of the $\alpha$-phage was established by McCloy (1951), who examined 171 strains of Bacillus anthracis and 244 other strains of other species of the genus Bacillus, by spotting a drop of the undiluted phage on a quarter plate spread previously with the bacterial strain to be tested. All the strains of $B$. anthracis were attacked by the phage, and only 2 of the 244 strains of other species were susceptible. These two were both $B$. cereus strains; another 54 strains of $B$. cereus were not attacked by the phage. Brown \& Cherry (1955) found that $\alpha$ phage lysed all of 41 naturally occurring rough strains of $B$. anthracis, 2 of 89 strains of $B$. cereus, and none of 134 strains of other Bacillus species.

The evidence presented in this paper indicates that it should be possible to identify Bacillus anthracis by means of specific bacteriophage within $\mathbf{7} \mathbf{h r}$. of sowing an agar plate with suspected material. 
I am grateful to Dr E. W. Meynell for a supply of $\alpha$ phage; to Dr A. J. H. Tomlinson for providing recently isolated strains of Bacillus anthracis; to Mr T. W. Pearce for valuable technical assistance; to Mr W. T. Bush for the photographs, and to Dr D. W. Henderson and Mr E. O. Powell for their interest and encouragement.

\section{REFERENCES}

Brown, E. R. \& Cherry, W. B. (1955). Specific identification of Bacillus anthracis by means of a variant bacteriophage. $J$. infect. Dis. 96, 34 .

McCloy, E. W. (1951). Studies on a lysogenic Bacillus strain. I. A bacteriophage specific for Bacillus anthracis. J. Hyg., Camb. 49, 114.

McCloy, E. W. (1958). Lysogenicity and immunity to Bacillus phage W. J. gen. Microbiol. 18, 198.

Pearce, T. W. \& Poweld, E. O. (1951). New techniques for the study of growing micro-organisms. J. gen. Microbiol. 5, 91.

\section{EXPLANATION OF PLATE}

Figs. 1-6. Transmitted light $16 \mathrm{~mm}$. objective. Magnification, $\times 150$.

Fig. 1. Two filaments of Bacillus anthracis, approximately at right angles to each other, on nutrient agar inoculated with spores and incubated $4 \mathrm{hr}$. at $37^{\circ}$.

Fig. 2. Microcolony of $B$. anthracis on nutrient agar. $6 \mathrm{hr}$. at $37^{\circ}$.

Fig. 3. Filament of $B$. anthracis on nutrient agar, $4 \mathrm{hr}$. at $37^{\circ},+1 \frac{1}{4 r}$. in presence of $\alpha$-phage. Lysis in the right-hand half of the filament, apart from one very short segment. The original position of the lysed filament is shown by a groove in the agar.

Fig. 4. Same filament as in fig. $3, \mathrm{hr}$. later. Lysis at four additional points.

Fig. 5. Microcolony of $B$. cereus on nutrient agar inoculated with spores and incubated $4 \mathrm{hr}$. at $37^{\circ}$.

Fig. 6. Microcolony of $B$. cereus on nutrient agar, $4 \mathrm{hr}$. at $37^{\circ},+1 \frac{1}{2} \mathrm{hr}$. in presence of $\alpha$ phage. Normal growth appearances; no lysis.

(Received 22 May 1959) 
Journal of General Microbiology, Vol. 21, No. 3
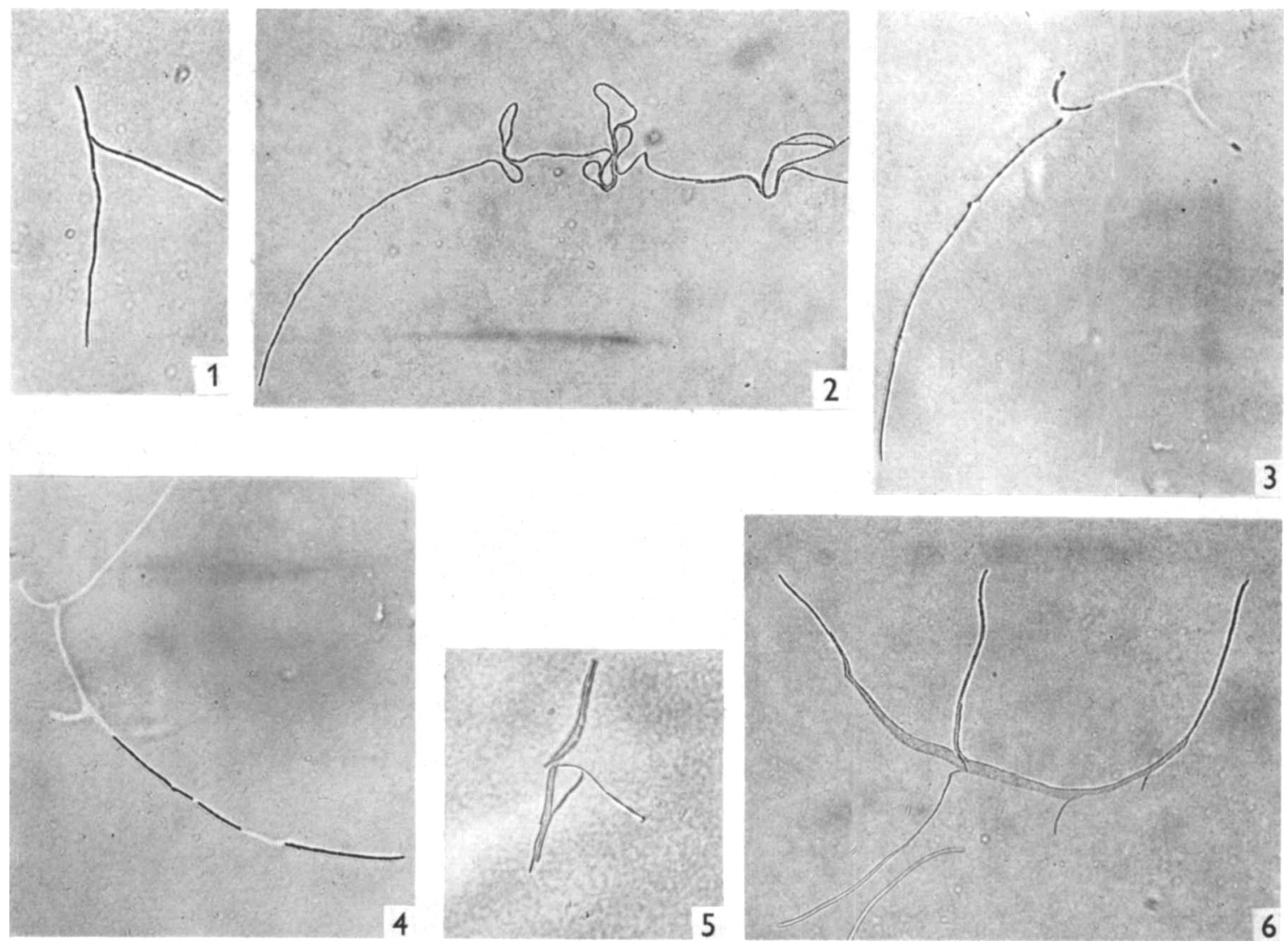

P. Chadwick-Rapid identification of B. anthracis. Plate 1 